\title{
DERECHOS SEXUALES Y REPRODUCTIVOS EN ARGENTINA: LOS PROYECTOS PARLAMENTARIOS REFERIDOS AL ABORTO ${ }^{1}$
}

\author{
SEXUAL AND REPRODUCTIVE RIGHTS IN ARGENTINA: \\ PARLIAMENTARY PROJECTS REFERRED TO ABORTION
}

\author{
Eugenia Zicavo ${ }^{2}$ \\ eugeniazicavo@yahoo.com \\ Julieta Astorino \\ astorinojuli@gmail.com \\ Lucas Saporosi ${ }^{4}$ \\ lucassaporosi@yahoo.com.ar
}

Fecha de recepción: 24 abril 2015 - Fecha de aceptación: 2 agosto 2015

\begin{abstract}
Resumen
En el presente artículo se examinan los proyectos de ley referidos al aborto, los cuales actualmente cuentan con estado parlamentario en Argentina (país donde dicha práctica es un delito penado con la cárcel, salvo contadas causales de no punibilidad). El objetivo es indagar, analizar y describir las consideraciones referidas a los derechos humanos y la salud, en tanto constituyen dos aspectos que aparecen reiteradamente en los diferentes proyectos, que a su vez remiten a una disputa por los significados en los discursos sociales circulantes con respecto al aborto. A partir de un relevamiento y análisis de documentos de acceso público (proyectos de ley presentados en las distintas Cámaras del Congreso Nacional), pondremos en perspectiva cultural el debate sobre la penalización/despenalización, a fin de comparar los diferentes proyectos de ley, la mayoría de los cuales se centra en la despenalización total y en una menor medida solo en modificaciones a los abortos no punibles, aunque también hay proyectos orientados a reforzar las restricciones vigentes. A la luz de los documentos, nos proponemos analizar los discursos sociales circulantes con respecto al aborto, observando las condiciones para el ejercicio de los derechos sexuales y reproductivos en el país.

Palabras claves: sexualidad, reproducción, derechos humanos, salud, interrupción voluntaria del embarazo.
\end{abstract}

$1 \quad$ Artículo derivado del proyecto de investigación en desarrollo R13-263 (2013-2015): "Los proyectos de ley referidos al aborto en la Argentina (1984-2013): historia, argumentos y perspectivas" del Programa de Reconocimiento Institucional de Investigaciones de la Facultad de Ciencias Sociales, Universidad de Buenos Aires, con sede en el Instituto de Investigaciones Gino Germani, Facultad de Ciencias Sociales, Universidad de Buenos Aires. Argentina. Directora: Dra. Eugenia Zicavo.

2 Doctora en Ciencias Sociales, docente en Sociología de la Cultura e Investigadora en el Instituto Gino Germani, Facultad de Ciencias Sociales, Universidad de Buenos Aires, Argentina.

3 Estudiante de la Carrera de Sociología, Facultad de Ciencias Sociales, Universidad de Buenos Aires, Argentina.

4 Licenciado en Sociología, Facultad de Ciencias Sociales, Universidad de Buenos Aires, Argentina. 


\begin{abstract}
This article examines the Congress Bills referred to abortion that currently have parliamentary state in Argentina (a country in which this practice is punishable with imprisonment, barring a few grounds for exemption). The objective is to investigate, analyze and describe some considerations related to human rights and health, two aspects that appear repeatedly in the different projects, which also refer to a dispute over the meaning of current social discourses regarding abortion. From a survey and analysis of public access documents (Congress Bills) the purpose is to put the criminalization/decriminalization debate in a cultural perspective. In order to do that, we compare the different bills, most of which focus on total decriminalization and to a lesser extent in modifications to the current legal abortions. Although there are also projects that aim at strengthening existing restrictions. We analyze, by studying the Congress Bills, the current social discourses relative to abortion, having in mind the conditions for the exercise of sexual and reproductive rights in the country.
\end{abstract}

Key words: Sexuality, Reproduction, Human Rights, Health, Voluntary Interruption of Pregnancy.

\title{
Introducción
}

Las condiciones para el cumplimiento efectivo de los derechos sexuales y reproductivos forman parte del debate público en la Argentina, que actualmente encuentra en el derecho al aborto un límite infranqueable para el pleno ejercicio de estos. Si bien desde el retorno de la democracia en 1983 ha habido avances en la materia, ${ }^{5}$ el aborto continúa tipificado en el Código Penal ${ }^{6}$ dentro de los delitos "contra las personas", que es penado con la cárcel. Según la legislación argentina vigente, ${ }^{7}$ el aborto es un delito excepto en las dos causales de no punibilidad contempladas: si se ha realizado con el fin de evitar un peligro para la vida o la salud de la madre (si este peligro no puede ser evitado por otros medios) y/o si el embarazo proviene de una violación o de un "atentado al pudor", cometido sobre una mujer "idiota o demente" (para el cual se requiere el consentimiento de su representante legal). Por una parte, el derecho de las mujeres para acceder a un aborto legal de acuerdo con las circunstancias previstas por el Código Penal tiene, sin embargo, interpretaciones encontradas. Por ejemplo, en lo relativo al criterio de "salud", este puede incluir tanto a la salud física como psíquica de la mujer embarazada (una lectura restrictiva contempla únicamente el aspecto físico o el riesgo de vida). Por otra parte, puede entenderse que el aborto legal es aplicable a todos los casos en que una mujer haya quedado embarazada como consecuencia de una violación, mientras que las interpretaciones restrictivas consideran no punibles solo los casos de violaciones a mujeres "idiotas o dementes". Estas interpretaciones contrapuestas también se ven reflejadas en los proyectos referidos al aborto presentados en el Senado y en la Cámara de Diputados del Congreso Nacional, la mayoría de los cuales apunta a realizar modificaciones con respecto a los abortos hoy considerados no punibles y a su despenalización sin restricciones hasta las 12/14 semanas de embarazo, aunque también hay proyectos que proponen endurecer las normas vigentes.

5 Entre ellos, el Programa de Salud Sexual y Procreación Responsable (2003), Ley de protección integral para prevenir, sancionar y erradicar la violencia contra las mujeres en los ámbitos en los que desarrollen sus relaciones interpersonales (2009), la Guía técnica para la atención integral de abortos no punibles (2010), el fallo de la Corte Suprema sobre abortos no punibles (2012).

6 A partir de la Ley 11179 de 1921.

7 El Código Penal, en su artículo 86, incisos 1 y 2 establece que: “... El aborto practicado por un médico diplomado con el consentimiento de la mujer encinta, no es punible: $1^{\circ}$ ) Si se ha hecho con el fin de evitar un peligro para la vida o la salud de la madre y si este peligro no puede ser evitado por otros medios; $2^{\circ}$ ) Si el embarazo proviene de una violación o de un atentado al pudor cometido sobre una mujer idiota o demente. En este caso, el consentimiento de su representante legal deberá ser requerido para el aborto". 
Las complicaciones por abortos son actualmente la principal causa de muerte de mujeres gestantes en Argentina y se calcula que se realizan cerca de medio millón de abortos clandestinos por año. ${ }^{8}$

De acuerdo con lo plateado y debido a que nos encontramos interesados en las normas sociales que, cristalizadas en las letras de la ley, condicionan la sexualidad y la reproducción al tiempo que son materia de deliberación pública y política, desde 2013 desarrollamos una investigación con sede en la Facultad de Ciencias Sociales de la Universidad de Buenos Aires, ${ }^{9}$ orientada a examinar los proyectos de ley referidos al aborto que se presentaron en ambas cámaras legislativas desde el regreso de la democracia. ${ }^{10}$ En el presente artículo presentamos algunos avances de dicha investigación, centrándonos en dos dimensiones: los derechos humanos y la salud, dos aspectos que aparecen reiteradamente en los proyectos, y que a su vez remiten a una disputa por los significados en los discursos sociales circulantes con respecto al aborto cristalizados en los proyectos de ley que actualmente tienen estado parlamentario. ${ }^{11}$

A la luz de los documentos, nos proponemos analizar los procesos políticos y los discursos sociales circulantes con respecto al aborto, a fin de dar cuenta de las condiciones para el ejercicio (o no) de los derechos sexuales y reproductivos en la Argentina.

\section{Metodología}

La metodología utilizada para nuestra investigación se basó en la técnica de análisis de documentos, especialmente en el relevamiento del contenido de los proyectos con estado parlamentario. En una primera instancia, se propuso una lectura detenida y analítica del corpus documental, atendiendo a las justificaciones, fundamentos y objetivos de cada proyecto. Confeccionamos una serie de grillas a partir de diferentes dimensiones y significantes (tales como salud, derechos humanos, derechos de la mujer, del feto, vida, madre, entre otros) buscando sistematizar la información y facilitar la comparación entre los documentos y sus dimensiones.

Este trabajo de grillado, sumado a la revisión bibliográfica sobre el tema, nos permitió realizar, por un lado, un análisis interno de los documentos, analizando cómo se expresan estas dimensiones en cada proyecto. Por otro lado, nos centramos en un análisis externo, procurando colocar cada proyecto de ley en su contexto, es decir, en el conjunto de circunstancias histórico-sociales de las que surgió. Para ello, nos propusimos comparar, describir y analizar las disputas en torno a esas dimensiones y sus posibles efectos en las representaciones sociales sobre el aborto.

Luego elaboramos una tipología que clasifica a los proyectos con base en una gradiente de derechos, que calificamos como: "progresista ampliatorio", "progresista moderado" y "restrictivo", siempre de acuerdo con el actual Código Penal argentino. Esta clasificación se fundamenta en el hecho de que la posibilidad de ejercer el derecho al aborto (de acuerdo con los proyectos de ley) reconoce una gradiente

8 Como se trata de una práctica realizada en la clandestinidad, no se cuenta con datos oficiales, pero diversos actores sociales acuerdan en que la cifra está entre los 350 mil y los 500 mil abortos anuales. Ver "Derechos Humanos en Argentina", Informe 2012, CELS (Centro de estudios legales y sociales) y "Estimación de la magnitud del aborto inducido en la Argentina" en Notas de población, nro. 87, CEPAL.

9 Proyecto R13-263: "Los proyectos de ley referidos al aborto en la Argentina (1984-2013): historia, argumentos y perspectivas" del Programa de Reconocimiento Institucional de Investigaciones de la Facultad de Ciencias Sociales, Universidad de Buenos Aires, Programación 2013-2015, con sede en el Instituto de Investigaciones Gino Germani, Facultad de Ciencias Sociales, Universidad de Buenos Aires. Directora: Dra. Eugenia Zicavo.

10 El poder legislativo ha presentado más de sesenta proyectos de ley referidos al aborto, sin lograr que ninguno llegue al recinto parlamentario para su debate y discusión.

11 Al momento de comenzar la investigación eran 16 los proyectos que tenían estado parlamentario (11 en Diputados y 5 en Senadores). Con el correr de los meses solo 5 mantienen dicho estado y en ellos centraremos nuestro trabajo, aunque haremos alusión a algunos de los proyectos que, habiendo perdido estado parlamentario, resultan enriquecedores para el análisis. 
que va desde la total despenalización del aborto hasta la restricción total, pasando por situaciones intermedias en las que dicho derecho sufre ciertas ampliaciones o excepcionalidades. Esta nos permitió hacer más claro el análisis y elaborar una síntesis conceptual de los puntos clave de cada proyecto.

El análisis documental realizado, situado en el ámbito de la investigación descriptiva, no solo pone el énfasis en el texto como fuente exclusiva de significación, sino que también recurre a las dimensiones socioculturales que le dan sentido a esos corpus legales y jurídicos. En este sentido, nos interesa poner en perspectiva cultural e histórica el debate sobre la penalización/ despenalización de la interrupción voluntaria del embarazo.

\section{Resultados}

A continuación presentamos los avances de la mencionada investigación. Para ordenar nuestra argumentación; en primer lugar, nos centraremos en la dimensión de los derechos humanos. Para ello, trabajaremos a partir de la clasificación de los proyectos antes referida, luego daremos cuenta de la tensión existente entre derechos, a partir de la consideración (o no) de los derechos de las mujeres (sexuales y reproductivos) como derechos humanos, y, por último, haremos mención a los tratados internacionales y legislaciones a las que refieren. Finalmente, abordaremos la dimensión salud y su expresión en los diferentes proyectos, de acuerdo, nuevamente, con la clasificación elaborada.

\section{Es una cuestión de derechos}

En el presente apartado analizaremos la dimensión de los derechos (de la mujer y/o del feto, según el caso) que aparecen en cada uno de los proyectos de ley con estado parlamentario, a partir de 3 ejes:

- El "espíritu del proyecto" en torno a un gradiente de derechos que nos permiten ubicar a cada uno en las siguientes categorías: "Progresista Ampliatorio", "Progresista Moderado" y "Restrictivo".

- La consideración (o no) de los derechos sexuales y reproductivos de las mujeres como derechos humanos (DDHH); y la existencia de contradicción-tensión (o no) entre derechos.

- Las citas, alusiones y usos de los mismos tratados internacionales/legislaciones para justificar y/o fundamentar cuestiones similares o hasta rotundamente opuestas.

\section{El "espíritu" de los proyectos de ley: "Progresista Ampliatorio", "Progresista Moderado" y "Restrictivo"}

Los proyectos a los que hemos denominado "progresistas moderados" generalmente proponen modificaciones que enmiendan al actual Código Penal, pero no modifican la cuestión de fondo en lo que respecta al debate sobre el aborto y su legislación. Los principales cambios se centran en lo que concierne a las causales de no punibilidad del aborto, incluyendo los casos de anencefalia fetal y los embarazos producidos por delitos contra la integridad sexual, sin realizar distinciones de acuerdo con si la mujer goza de sus facultades mentales o no. Esta clase de proyectos de ley es vista por ciertos autores como un refuerzo de la interpretación conservadora del Código Penal, tal como afirma Maffia (2006, p.5) al decir que "algunos legisladores progresistas han presentado como una innovación proyectos para despenalizar el aborto en casos de violación, lo cual significa que participan de la misma lectura restrictiva del Código Penal". Esto es interesante para pensar y hacer más claras las interpretaciones y disputas que hay alrededor del aborto y sus debates y a su vez para ver los límites que presentan en la realidad, las categorías analíticas pensadas. Dentro de esta tipología ubicamos dos proyectos presentados en la Cámara de Diputados (2502 D 2012 y 2503 D 2012) que tienen puntos y argumentos en común, pues ambos fueron presentados por la misma legisladora. 
Entre los proyectos que denominamos "progresistas ampliatorios" se encuentran los proyectos 3067 D 2013, 0041 D 2014 y 2249 D 2014. Estos proponen ampliaciones a las actuales causas de aborto no punible, enmendando al actual Código Penal, y plantean -entre otras cosas- la despenalización del aborto y la derogación de los artículos que lo tipifican como delito. Se contempla la libertad de las mujeres a decidir sobre sus cuerpos, ya que según la letra de uno de los proyectos (2249 D 2014):

El derecho a decidir no sólo se circunscribe al derecho a la interrupción de un embarazo, sino que incluye también la posibilidad de decidir la maternidad. Es reconocer a las mujeres como sujetos sociales, como ciudadanas y agentes morales capaces de decidir si desean o no ser madres, el número de hijos y el espacio entre los nacimientos.

Los proyectos que tipificamos como restrictivos ${ }^{12}$ proponen la derogación de las actuales causas de aborto no punible, restringiendo aún más el acceso y cumplimiento de los derechos sexuales y reproductivos de las mujeres. Dentro de esta tipología se encuentran los proyectos 0856 D 2009 y 0062 S 2009. Ambos proponen modificaciones al artículo 86 del Código Penal, pero no para ampliar los casos de aborto no punible, sino todo lo contrario: plantean suprimir el inciso $2 .^{\circ}$ del mencionado artículo (los casos de violación a mujeres idiotas o dementes) por considerar que las excepciones dispuestas en este son "inconstitucionales", pues, según plantea el texto del proyecto 0062 S 2009, "violan el derecho a la vida de la persona concebida sin que corra riesgo ninguna otra vida ni que la salud de alguien se vea perjudicada". Es decir, presupone que la salud psíquica de una mujer embarazada producto de una violación no será en absoluto vulnerada si es obligada a llevar adelante una maternidad forzada. El proyecto 0856 D 2009 incluso plantea que el estado les otorgue un subsidio económico a las mujeres "si su situación es de vulnerabilidad, indigencia, pobreza, exclusión o riesgo social", además de aclarar lo que denomina "aborto indirecto", aquel realizado en caso de que corra peligro la vida de la mujer embarazada, única circunstancia en la que ambos proyectos contemplan la no punibilidad del procedimiento.

\section{Derechos humanos y derechos sexuales y reproductivos}

En este apartado analizaremos cada proyecto según considere (o no) a los derechos sexuales y reproductivos de las mujeres como DDHH, contemplando la existencia de contradicción-tensión (o no) entre derechos.

El proyecto 2502 D 2012 propone ampliar los criterios de no punibilidad para los casos en que se busca evitar un peligro para la vida o salud de la mujer, en los casos de abuso sexual con acceso carnal (con presentación de la denuncia policial o penal, y antes de las 12 semanas) y en los casos de anencefalia. Mientras que el proyecto 2503 D 2013 establece un plan de acción integral para la salud de la mujer en los casos de abortos no punibles, concebidos como aquellos realizados a víctimas de abuso sexual (con denuncia penal o policial) y en los casos de riesgo de vida para la vida o salud de la mujer. Este proyecto se detiene en cuestiones técnicas y procedimentales con respecto a la realización de los abortos no punibles. Ambos proyectos son autoría de la misma legisladora, por lo que realizaremos su análisis en conjunto.

Los derechos humanos de las mujeres quedan, en estos proyectos, subsumidos a los derechos del "niño por nacer" (según palabras del proyecto), donde la supuesta tensión entre ambos derechos se dirime en favor del feto, salvo en las contadas excepciones aludidas en el proyecto.

Estos proyectos de carácter restrictivo plantean que:

El Estado implemente políticas de apoyo a la maternidad, para que ante los embarazos no deseados, inclusive en caso de violación, las mujeres no elijan el aborto como una solución, sino que puedan analizar la posibilidad de continuar el embarazo con el acompañamiento sustentable y eficaz de programas gubernamentales, sea para llevar adelante la crianza por parte de su progenitora o para su adopción.

12 En el caso de los proyectos que consideramos restrictivos, si bien han perdido recientemente su carácter parlamentario, hemos decidido incluir algunos en el análisis a efectos de dar cuenta en este artículo de la matriz de sentido en la cual inscriben sus argumentaciones y propuestas. 
Aquí vemos cómo se refuerza la matriz de identidad mujer igual madre, al afirmar que si bien se contemplan los casos de violación para realizarse un aborto, buscan fortalecer ante todo la maternidad, incluso aquella violenta o forzada. Por último, se expresa que en los casos de violación se debe presentar la denuncia policial o penal, porque si esto no se hiciera implicaría la despenalización del aborto, considerando a su vez que la propia denuncia constituye una función preventiva. Con esto no contemplan que, si bien se trata de una acción legítima, promover una acción penal también implica en muchos casos una re victimización de la mujer, porque como afirma MacKinnon (2014):

\footnotetext{
Las mujeres sentimos miedo y desesperación al enfrentarnos a la policía, los hospitales y el sistema judicial. Las mujeres no sólo creemos que la policía no nos creerá y que los médicos nos tratarán de manera degradante, sino también que, cuando acudamos a la justicia, el incidente no será visto desde nuestro punto de vista (...) el miedo a ser maltratadas no es un invento de la imaginación de las mujeres. Es el resultado directo de la forma como nosotras hemos sido tratadas (Pp. 310).
}

Por su parte, el proyecto 3067 D 2013 establece la derogación de los artículos 85 y 88 del Código Penal que tipifican al aborto como delito, y propone modificaciones al art. 86, como la no punibilidad para los casos de delitos contra la integridad sexual y en el caso de que sea solicitado por la mujer embarazada antes de las doce semanas de gestación. Con ello busca terminar con la prolongación de la violencia contra las mujeres al obligarlas a continuar con un embarazo impuesto por la fuerza, violencia ejercida desde la justicia y el ejercicio de las leyes, que muchas veces limitan su autonomía y las re victimizan. En sus postulados prima el derecho a la integridad física de la mujer y a decidir sobre el propio cuerpo, considerando la maternidad como opcional, como decisión. Así se cuestiona la matriz de pensamiento que concibe a las mujeres en tanto madres y cuidadoras, como lo explicita Pateman (1996, p.96) al afirmar que "el patriarcalismo recurre a la naturaleza y al supuesto de que la función natural de las mujeres consiste en la crianza de los hijos, lo que prescribe su papel doméstico y subordinado en el orden de las cosas". Es decir, que concibe a las mujeres en tanto autónomas y con pleno ejercicio de sus derechos, cuestionando los imperativos culturales que refuerzan y reproducen el modelo obligatorio de mujer-madre.

En relación con el proyecto anterior, el 0041 D 2014 establece que el aborto practicado por un médico diplomado y con el consentimiento de la mujer embarazada no es punible si se realiza para evitar un peligro para la vida o salud de la mujer, si el embarazo es producto de un delito contra la integridad sexual, si se diagnosticó la inviabilidad de la vida extra uterina del feto y si se realiza durante las 12 primeras semanas de gestación. En sus fundamentos hay alusiones a tratados internacionales y a sus recomendaciones en materia de salud sexual y reproductiva, y a los derechos de las mujeres y el respeto a los mismos. Aquí es donde queremos poner el foco de atención. El proyecto afirma que:

El debate sobre el tratamiento del aborto debe realizarse en el marco de la consideración de los derechos humanos, en el que están involucrados el respeto a la autonomía, a la salud integral, a la dignidad, entre otros. Los derechos humanos de las mujeres requieren la consideración de los derechos acordes a su especificidad sexual y reproductiva.

Creemos que en esta afirmación está una de las claves para entender la problemática del aborto y la reivindicación del mismo como derecho. Las mujeres son concebidas como sujetos de derecho, activos, autónomos. Por otro lado, según este proyecto, la amenaza de prisión para quien que se realizó un aborto constituye una sanción injusta que pone a la mujer en la situación de tener que elegir entre un aborto clandestino realizado en condiciones que ponen en peligro su vida y salud, o someterse en el caso de violación a una maternidad violenta, impidiendo que puedan acceder a la opción de realizarse un aborto en condiciones seguras y dignas se refuerza el imperativo de mujer igual madre, lo que imposibilita pensar a la mujer como sujeto de derecho, como ciudadana activa y con autonomía sobre su propio cuerpo, sin que quede subsumida al rol de procreadora y cuidadora. 
La perspectiva que orienta este proyecto expresa la consideración de los derechos (sexuales y reproductivos) de las mujeres como derechos humanos, considerando la prohibición del aborto y su penalización como un atentado a estos; enmarcando el debate sobre el aborto dentro de la discusión de los derechos humanos.

En el caso del proyecto 2249 D 2014, la apelación a los derechos humanos también es recurrente, tanto en las alusiones a convenciones y tratados internacionales, como en los fundamentos del texto. Como se expresa en la letra del proyecto:

\begin{abstract}
Consideramos que los derechos sexuales y los derechos reproductivos son derechos humanos y deben ser reconocidos como derechos básicos de todas las personas. Para eso, es necesario garantizar el acceso universal a los servicios públicos que los sostienen. Nuestro lema -así como nuestro trabajo de años- es integral: "educación sexual para decidir, anticonceptivos para no abortar, aborto legal para no morir". Esto implica la exigencia hacia el Estado de realizar modificaciones en los sistemas de Educación, Salud y Justicia, y también, por supuesto propiciar en la sociedad profundos cambios culturales. (...) Nos basamos en la integralidad, interdependencia e indivisibilidad de los derechos humanos.
\end{abstract}

Este proyecto contempla el derecho al aborto como un derecho que integra un conjunto más amplio, el de los derechos reproductivos, y afirma que éstos deben ser reconocidos como DDHH, basándose en el principio de integralidad, interdependencia e indivisibilidad de los mismos. El derecho a decidir no solo incumbe a la interrupción del embarazo sino que incluye también el derecho a la opción por la no-maternidad. Es reconocer a las mujeres como sujetos sociales, como ciudadanas y agentes morales; tal como se enuncia en el proyecto:

\footnotetext{
Argentina como país signatario de pactos y convenciones de derechos humanos y sus protocolos facultativos, que forman el corpus jurídico de este derecho, está obligada a garantizar a las mujeres los siguientes derechos: a la vida, al máximo nivel posible de salud física y mental, a la igualdad y no discriminación, a la intimidad y a la autonomía reproductiva, a la libertad, a verse libre de tratos crueles inhumanos y degradantes, a la libertad de conciencia y religión, entre otros. La prohibición del aborto y la falta de acceso a procedimientos seguros, de calidad y gratuitos es una violación a estos derechos.
}

A contramano de lo expresado anteriormente, los dos proyectos de carácter restrictivo no consideran a los derechos sexuales y reproductivos de las mujeres como DDHH, sino que ponen en consideración y con mayor énfasis los derechos del feto, entendiéndolos como "derechos del niño por nacer". Los derechos sexuales reproductivos de la mujer se presentan como una cuestión subsidiaria, y su derecho a la autonomía -un componente básico del derecho a la libertad- es enmendado por propuestas de "ayuda" o "acompañamiento" (emocional y/o económico) que la mujer debería recibir a la hora de encarar una maternidad forzada.

\title{
Los tratados internacionales y el debate sobre el aborto
}

En el presente apartado nos centraremos en los usos que los diferentes proyectos de ley hacen de los mismos tratados internacionales/legislaciones para justificar, fundamentar cuestiones similares o hasta rotundamente opuestas.

En líneas generales todos los proyectos de carácter "progresista ampliatorio" y "progresista moderado" citan en sus fundamentos casi los mismos tratados internacionales, entre ellos, el Consejo de DDHH de la ONU, la Conferencia de la Mujer, la Conferencia Internacional sobre Población y desarrollo, la Convención contra todas las formas de discriminación sobre la mujer, la Conferencia Internacional de DDHH. Estos son citados en las fundamentaciones de los proyectos, en especial las recomendaciones que dichas instancias internacionales han elevado para que el aborto sea abordado como una problemática de salud pública, reconociendo los derechos sexuales y reproductivos de las mujeres como DDHH, y a su vez, para incorporar políticas públicas que los contemplen como tales. 
Estos organismos instan a los gobiernos a incrementar su compromiso con la salud de las mujeres, a ocuparse de las consecuencias de los abortos realizados en condiciones no adecuadas como un importante problema de salud pública y a reducir el recurso del aborto mediante la prestación de más amplios y mejores servicios de planificación familiar. Además, recomiendan revisar las leyes que contemplan medidas punitivas contra las mujeres que han tenido abortos ilegales y específicamente han puesto en consideración la necesidad de que se revise la legislación argentina sobre el aborto.

Resulta interesante el uso que se hace de la Convención de los Derechos del Niño, que es citada tanto por los proyectos restrictivos como progresistas, en argumentaciones de carácter completamente opuesto. Por un lado, es citada en los proyectos que proponen la total despenalización del aborto en lo referente a los casos de violación de niñas menores de 14 años, donde se afirma que se debe velar por los intereses de la niña y se deben respetar sus decisiones, "en pleno goce de sus derechos y garantías consagrados en la Convención Internacional de los Derechos del niño". Pero a su vez lo utilizan los dos proyectos de carácter restrictivo para plantear los derechos del niño por nacer, para que se respete, según ellos, el derecho a la vida.

Como aparece expresado en el proyecto 0062-S-2009:

La Convención de los Derechos del Niño, en su art. 1 establece que se entiende por niño todo ser humano menor de 18 años de edad. En esto la República Argentina aclara que debe interpretarse en el sentido de que se entiende por niño todo ser humano desde el momento de la concepción y hasta los 18 años de edad. La Convención Americana sobre DDHH establece que toda persona tiene derecho a que se respete su vida.

De ese modo afirman que aquellos tratados aprobados por el Congreso y la Constitución Nacional están por encima del Código Penal y que, en el caso de que entraran en contradicción, tienen validez por sobre éste.

Retomando lo referido a los tratados y legislaciones internacionales a los que aluden los distintos proyectos, es curioso como los derechos humanos son invocados tanto para justificar la necesidad de una ley de aborto legal, seguro y gratuito como para restringir aún más las pocas circunstancias que actualmente son contempladas como abortos no punibles. Como afirman Faúndes y Barzelatto (2011, p. 166) "el aborto genera una tensión, al menos, entre dos derechos humanos, el derecho de la vida del embrión o feto, y la autonomía de la mujer, un componente básico del derecho a la libertad". Como no hay orden jerárquico entre los derechos humanos, cuando hay un conflicto se apela a la interpretación. En el debate en torno al aborto, nos parece fundamental analizar cuáles son los significantes utilizados para referirse a cada uno de los sujetos y términos involucrados, ya que se trata de un terreno de disputas y de imposición de interpretaciones. Los proyectos de ley que apuntan a despenalizar el aborto y a reglamentarlo dentro de la ley no encuentran contradicción alguna entre defender los DDHH y bregar por el aborto legal. Del mismo modo, los proyectos que buscan ser aún más restrictivos que la norma vigente, que plantean que incluso los abortos realizados a mujeres dementes violadas deberían ser penados por la ley, no encuentran contradicción entre defender los DDHH y la imposición de una maternidad forzada a las mujeres.

Por eso es necesario detenerse en el modo en que los proyectos de ley argumentan la necesidad de modificar la legislación existente. Porque aludir al aborto como derecho humano, además de contemplarlo dentro del derecho a la salud, es también incluir a un derecho exclusivo de las mujeres dentro del universal de humanidad que tanto tiempo les fue negado (tanto en las leyes como en el lenguaje).

\section{Los criterios de salud}

En este apartado buscamos poner de relieve los criterios de salud que sustentan los diferentes proyectos analizados. En general, los proyectos de carácter "Progresista ampliatorio" y "progresista moderado" hacen referencia a una concepción integral de la salud de la mujer en tanto persona portadora de 
derechos, por lo que contemplan el plano psíquico, físico y sus consecuencias sociales. En ellos aparecen significantes tales como "salud integral", "salud reproductiva", "derechos sexuales y reproductivos".

Por ejemplo, en el proyecto 2249 D 2014 (progresista ampliatorio), la "salud" no solo tiene que ver con la dimensión corporal y biológica de este derecho, sino también con sus efectos sociales y culturales, en términos de decisión y autonomía. Como se afirma en el proyecto:

El derecho a decidir no sólo se circunscribe al derecho a la interrupción de un embarazo, sino que incluye también la posibilidad de decidir la maternidad. Es reconocer a las mujeres como sujetos sociales, como ciudadanas y agentes morales capaces de decidir si desean o no ser madres, el número de hijos y el espacio entre los nacimientos.

Es decir, preserva el derecho de las mujeres a planificar su maternidad en el momento que así lo deseen.

Por su parte, el Proyecto 0041-D-2014 expone que "en todos los casos las mujeres deberían tener acceso a servicios de calidad para tratar complicaciones derivadas de abortos. Se deberían ofrecer con prontitud servicios de planificación de familia, educación y asesoramiento post aborto que ayuden a evitar la repetición de los mismos". A su vez, al permitir la realización del aborto en casos de anencefalia del feto se refiere exclusivamente a la salud psicológica de la mujer, y alude a la "catástrofe psíquica" de "sobrellevar el crecer muriendo de ese ser vivo, proceso que se desenvuelve dentro de ella. Un proceso que conduce al progresivo deterioro de la capacidad de humanizarse que padece ese feto, al que, sin embargo, ella humanizó al hacerlo su hijo." Es decir, sustenta una concepción integral de la salud, considerando no solo los aspectos físicos, sino también los psíquicos.

En la misma línea, el proyecto 3067 D 2013 expone que es necesario ocuparse de los efectos que generan en la salud de las mujeres los abortos realizados en condiciones no adecuadas, como parte de un problema de salud pública. Además, pone en consideración la necesidad de que se presten mejores y más servicios de planificación familiar. Este proyecto también tiene una concepción integral de la salud, al considerar los factores sociales que intervienen en la problemática de aborto.

Los proyectos 2502 y 2503 D 2012 (progresistas moderados) hacen referencia a la integralidad del concepto de salud, cuando el aborto proviene de una violación, de la comprobación médica de la inviabilidad de vida extrauterina del feto o cuando la vida de la mujer corre peligro. Cuando ello ocurre, el concepto de salud se concibe desde sus múltiples dimensiones. Se prevé la atención médica y psicológica de las mujeres que se realicen un aborto (en el marco de las excepciones contempladas por los proyectos), se establecen las condiciones en que deben realizarse esos abortos, además del derecho a la información y al consentimiento de la mujer para realizar el proceso. Por último, se dispone que la entidad de aplicación y control sea el Ministerio de Salud de la Nación.

En relación con los proyectos de carácter regresivo, el 0856 D 2009 solo contempla la no punibilidad del aborto en caso de que este hubiera sido practicado de manera "indirecta", es decir, que haya sido provocado por el médico sin intención, con el fin de salvar la vida de la madre. Es decir, solo entiende a la salud en términos exclusivamente físicos pero no tiene en cuenta la salud integral al considerar punibles incluso los abortos realizados a mujeres embarazadas producto de una violación, es decir, sin tener en cuenta los factores psíquicos.

Según el proyecto 0062 S 2009, también de carácter regresivo, el aborto debe ser punible incluso en casos de violación a mujeres dementes. Este proyecto no concibe ninguna dimensión integral relativa a la salud de las mujeres dado que su atención está puesta exclusivamente en "la vida por nacer" y se ampara en el artículo 14 bis de la Constitución Nacional que "dispone la protección integral de la familia. Una madre con un hijo en su seno es parte esencial de una familia que merece la protección integral". Según afirma el proyecto: "El niño concebido es el fruto y efecto de la vida familiar que como legisladores de la Nación tenemos la obligación, también por este artículo, de proteger".

El proyecto reproduce acríticamente la matriz socio-cultural que tiende a naturalizar las relaciones sexo-género y a las instituciones surgidas de ellas (en este caso, la familia) y la mera figura de la 
mujer embarazada habilita en sus presupuestos toda una cadena de significantes (en este caso, "madre", "hijo" y "familia") como si todos los embarazos fueran el resultado de la "vida familiar" (no contempla a las madres solteras, ni a los embarazos producto de violaciones) y como si el ejercicio libre de la sexualidad estuviera exclusivamente enmarcado dentro de dicha institución.

\section{Conclusiones}

En Argentina, el debate por la despenalización del aborto ha buscado varias oportunidades en el Congreso y sin embargo ninguna de las iniciativas logró prosperar y fueron perdiendo estado parlamentario. Se trata de un tema del orden de la salud pública y una cuestión de justicia social (las mujeres con menores recursos son las más afectadas por las consecuencias de abortos mal realizados) y de derechos (a la integridad física, a la autonomía individual, al control sobre el propio cuerpo) que encuentran -o no- expresión en los distintos proyectos a nivel parlamentario.

La discusión pública con respecto a la despenalización y legalización del aborto resulta fundamental y urgente en términos de salud pública, al relacionarse directamente con el derecho a la autonomía reproductiva, al máximo nivel posible de salud física y mental, a la igualdad y no discriminación, a la libertad de conciencia y religión, entre otros. Ya que, como plantea Bellucci (2014):

Quienes nos pronunciamos a favor de la despenalización del aborto nos estamos pronunciando a favor de ese espacio íntimo de lo que puede atribuirse por derecho propio una mujer. Quienes legislan a espaldas de la realidad castigan con su indiferencia a aquellas que sí decidieran sobre su propio cuerpo. Ejercen de un modo explícito y abierto la violencia sobre ellas (Pp. 322).

El proyecto de ley propiciado por la Campaña Nacional por el Derecho al aborto (conformada en 2005), firmado por legisladores de distintos partidos y signos políticos, que apunta a que las mujeres puedan decidir libremente la interrupción de su embarazo durante las primeras doce semanas del proceso gestacional, no llegó nunca a ser debatido en el recinto (a pesar de que los firmantes pertenecen a distintas fuerzas políticas). Se trata de un reclamo de larga data, o como dice uno de los slogans de la Campaña "una deuda de la democracia". La reticencia por tratar el tema a nivel parlamentario en nuestro país da cuenta, a la luz de los documentos, tanto de los procesos políticos como de la permeabilidad de los discursos y mandatos sociales circulantes respecto al aborto, a la maternidad, a las mujeres y a sus derechos sexuales y reproductivos. En estos discursos, la maternidad y la femineidad se presentan como un evento natural en la vida de las mujeres (Garay, 2008).

La problematización y discusión sobre derechos sexuales y reproductivos fue ganando visibilidad en la agenda pública argentina durante los últimos veinte años, con ciertos logros en materia del cuidado de la salud sexual y reproductiva. Sin embargo, el aborto sigue siendo un terreno de disputa en el marco de lo legal y los proyectos de ley presentados en ambas cámaras legislativas son emergentes de distintas matrices significantes. La ampliación de la ciudadanía sexual tiene que ver directamente con la posibilidad efectiva de disociar sexualidad y reproducción sin por ello poner en riesgo la vida. En el espacio público político, el debate sobre sexualidad y reproducción evidencia argumentos atravesados por concepciones encontradas sobre el cuerpo, el rol social de las mujeres y diversos consensos de orden cultural.

Considerando la relación y significación atribuida al espacio público (y privado) en relación con la ciudadanía y las mujeres, entendemos la separación entre sexualidad y reproducción como parte fundamental de una ciudadanía sexual plena en la cual las mujeres puedan decidir libre y responsablemente el número y espaciamiento de los hijos y creemos que, como plantea Brown (2011, p. 23), "la consideración de la cuestión dentro del orden de lo privado obstaculizó, durante largo tiempo, su debate público y político.”

Como hemos advertido en el presente artículo, proyectos de distinto signo aluden a los mismos tratados internacionales para justificar posiciones disidentes, como por ejemplo la Convención 
Internacional de los Derechos del Niño. Sin embargo, los proyectos de carácter restrictivo, con solo apelar a la norma constitucional, parecen quedar liberados de otro tipo de argumentación. En sus fundamentos, se amparan en el lenguaje legítimo, aquel que emana de la Constitución, mientras que los proyectos progresistas (en general) encuentran más dificultades para dar a sus fundamentos un marco de legitimidad dentro del orden socio-legal vigente.

En este sentido, podemos pensar el mantenimiento de la punibilidad del aborto y su tipificación como delito en tanto un modo de sostener un determinado orden social respecto al aborto y a la sexualidad femenina y, a su vez, pensar esta clase de proyectos como formas de cuestionar ese orden y desnaturalizarlo.

\section{Referencias}

Bellucci, Mabel (2014). Historia de una desobediencia. Aborto y feminismo. Buenos Aires: Capital Intelectual.

Brown, Josefina (2011). "Derechos (no) reproductivos y sexuales en los bordes entre lo público y lo privado. Algunos nudos del debate en torno a la democratización de la sexualidad" en Petracci, Mónica (comp.) Derechos sexuales y reproductivos. Teoría, política y espacio público. Buenos Aires: Teseo.

CELS (2012) Derechos Humanos en Argentina. Informe 2012. Buenos Aires: Siglo XXI.

Faúndes, Aníbal y Barzelatto, José (2011). El drama del aborto. En busca de un consenso. Buenos Aires: Paidós.

Garay, Ricardo (2008) "El destino de ser madres: ideología de la maternidad como soporte discursivo de las nuevas tecnologías reproductivas" en Tarducci, Mónica (coord.) Maternidades en el siglo XXI. Buenos Aires: Espacio Editorial.

MacKinnon, Catherine (2014). Feminismo inmodificado. Discursos sobre la vida y el derecho. Buenos Aires: Siglo XXI.

Maffía, Diana (2006). “Aborto no punible: ¿Qué dice la ley argentina?” en Susana Checa (comp.) Realidades y coyunturas del aborto. Entre el derecho y la necesidad. Buenos Aires: Paidos. Extraído de http://dianamaffia.com.ar/archivos/Aborto-no-punible.-Qu\%C3\%A9-dice-la-ley.pdf

Mario, Silvia y Pantelides, Edith Alejandra (2009) "Estimación de la magnitud del Aborto Inducido en Argentina" en Notas de población, nro. 87, CEPAL.

Pateman, Carole (1996). "Críticas feministas a la dicotomía público/privado.” en Carme Castells (comp.) Perspectivas feministas en teoría política. España: Paidos. 
